\title{
COMPARAÇÃO ENTRE OS MÉTODOS LABORATORIAL E PORTÁTIL NA ANÁLISE DA GLICEMIA EM FELINOS COM AMOSTRAS DE SANGUE VENOSO CENTRAL E CAPILAR
}

\section{COMPARISON BETWEEN THE LABORATORY AND PORTABLE METHODS IN THE GLYCEMIC ANALYSIS IN CATS WITH CENTRAL VENOUS AND CAPILLARY BLOOD SAMPLES}

\author{
Yonara Silva Garcia de Oliveira ${ }^{1}$ \\ Jair Duarte da Costa Júnior² \\ André Santos-Leonardo ${ }^{3}$ \\ Kamila Santos de Morais ${ }^{3}$ \\ ${ }^{1}$ Graduanda em Medicina Veterinária da Universidade de Brasília, Brasília, DF, Brasil - \\ yonaragarcia@yahoo.com.br \\ 2Professor Doutorada Universidade de Brasília, Brasília, DF, Brasil \\ 3Pós-graduandos da Universidade de Brasília, Brasília, DF, Brasil
}

\section{Resumo}

Objetivou-se comparar a eficácia do método portátil de mensuração glicêmica com o método laboratorial de referência, utilizando-se amostras de sangue venoso central e de sangue capilar de 20 felinos. Considerou-se como valor padrão de referência o obtido pelo método laboratorial a partir de amostra de sangue venoso central. Outros dois valores foram obtidos por glicosímetro portátil, sendo utilizada amostra do mesmo sangue venoso central e outra amostra a partir de sangue capilar. Para os 20 felinos, os resultados glicêmicos verificados pelo método laboratorial tiveram média de $80 \mathrm{mg} / \mathrm{dL}$; e os resultados constatados pelo glicosímetro, média de 84,1mg/dL com sangue venoso central e 73,1mg/dL com sangue capilar. As médias glicêmicas obtidas pelo glicosímetro com ambas as amostras de sangue em relação à alcançada pelo método laboratorial resultaram em variações inferiores à máxima instituída (20\%) por órgãos reguladores de glicosímetros e por entidade de padronização, bem como a variação entre as médias glicêmicas obtidas pelo aparelho com as distintas amostras de sangue. $\mathrm{Na}$ análise comparativa houve diferença significativa entre os métodos $(\mathrm{p}<0,05)$. Concluiu-se que o glicosímetro portátil é eficaz para mensuração da glicemia em felinos, tanto a partir de amostras de sangue venoso central como de sangue capilar.

Palavras-chave: gato; glicose; glicosímetro; mensuração.

\begin{abstract}
The objective of this study was to compare the efficacy of portable blood glucose measurement method in relation to the laboratory reference method, using central venous and capillary blood samples from 20 cats. The value obtained by the laboratory method using central venous blood sample was considered as the standard reference value. Other two values were obtained from the
\end{abstract}


portable blood glucose meter, in which a sample from the same central venous blood and another one sample from capillary blood were used. For the 20 cats, the blood glucose results obtained by the laboratory method had an average of $80 \mathrm{mg} / \mathrm{dL}$; and the results found by the glucometer had an average of $84.1 \mathrm{mg} / \mathrm{dL}$ with central venous blood and of $73.1 \mathrm{mg} / \mathrm{dL}$ with capillary blood. The glycemic averages obtained by the glucometer with both blood samples compared to that achieved by the laboratory method resulted in variations below the maximum established (20\%) by associations that regulate portable glucometers and by the organization for standardization, as well as the variation between the glycemic averages obtained by the glucometer using different blood samples. In the comparative analysis, there was no significant difference between the methods $(\mathrm{p}<0.05)$. In conclusion, the portable glucometer is effective in measuring blood glucose in cats, both from central venous blood and capillary blood samples.

Keywords: cat, glucometer, glucose, measurement.

Recebido em: 28 julho 2014

Aceito em 23 janeiro 2015

\section{Introdução}

A determinação da glicemia é essencial para o diagnóstico e controle de qualquer condição que afete a homeostase glicêmica. Endocrinopatias como o diabetes melito exigem constante monitoração dos níveis de glicemia a fim de se evitarem complicações, permitindo adequações na dieta e na terapia dos pacientes ${ }^{(1-3)}$.

A concentração sanguínea de glicose é obtida como referência pelo método enzimáticocolorimétrico, também chamado de metodologia de Trinder ${ }^{(4,5)}$, através do espectofotômetro de luz, um equipamento de bancada laboratorial. O princípio da reação é a oxidação da glicose da amostra sob a ação catalisadora da enzima glicose-oxidase presente no reagente. O produto final é uma substância vermelha cuja intensidade da cor é proporcional à concentração de glicose na amostra ${ }^{5}$. Esse método requer muito volume de sangue para análise, além de tempo e custo para a obtenção dos resultados ${ }^{(4)}$.

Para facilitar as mensurações glicêmicas, surgiram na década de 70 os medidores portáteis de glicose, fabricados para que pessoas diabéticas pudessem monitorar sua glicemia ao longo do dia. Essa tecnologia é utilizada em pacientes caninos com relativa segurança e praticidade ${ }^{(5,6-11)}$. Dentre os vários benefícios que os glicosímetros oferecem em relação aos métodos laboratoriais destaca-se o fato de serem aparelhos pequenos, portáteis, de fácil manuseio e que necessitam de pouca quantidade de amostra sanguínea (geralmente de 1 a $5 \mu \mathrm{L}$ ). Rapidez na obtenção dos resultados e menor custo também são fatores destacados ${ }^{(6,7)}$. Tais aparelhos utilizam fitas reagentes descartáveis e funcionam por tecnologias de fotometria de reflexão ou de amperometria eletroquímica ${ }^{(12)}$.

Instituições reguladoras de glicosímetros portáteis, como a American Diabetes Association (ADA), preconizam que todos os aparelhos apresentem variação inferior a $15 \%$ em relação aos métodos bioquímicos laboratoriais na concentração da glicose plasmática para valores entre 30 e $400 \mathrm{mg} / \mathrm{dL}$. A Food and Drug Administration (FDA) recomenda que essa variação seja de até $20 \%$ para a mesma concentração ${ }^{(14)}$. De acordo com a Internacional Organization for Standardization (ISO), os valores glicêmicos obtidos pelos aparelhos portáteis não podem variar mais que $20 \%$ dos valores 
encontrados pelo método laboratorial padrão ${ }^{(10,11,13)}$.

Uma vez que vários estudos mostram que em cães existe confiabilidade no uso dos medidores portáteis, questionamentos ainda são feitos a cerca da acurácia desta tecnologia no monitoramento da glicemia em pacientes felinos, cujos estudos são escassos. Diante do exposto, torna-se relevante a comparação entre o método portátil de mensuração da glicemia e o método laboratorial de referência e, adicionalmente, a comparação dos resultados glicêmicos provenientes de amostras de sangue venoso central e sangue capilar.

\section{Material e Métodos}

Foram obtidas amostras de sangue de 20 felinos, machos e fêmeas, independente de raça e idade, não acometidos por doenças hipo ou hiperglicemiantes, todos em jejum mínimo de seis horas. Para as amostras de sangue venoso central, as coletas foram realizadas por punção da veia jugular externa com auxílio de agulha hipodérmica calibre 25 x $0,7 \mathrm{~mm}$ e seringa de $3 \mathrm{~mL}$, descartáveis $\left(\right.$ Embramac $\left.^{\circledR}\right)$. Após contenção manual e antissepsia com álcool $70 \%$ na região ventral do pescoço, puncionou-se a veia jugular para obtenção de sangue venoso central. Todo o procedimento foi realizado de maneira rápida e delicada para se evitarem alterações pelo estresse.

Após a colheita, as amostras de sangue foram acondicionadas em tubos contendo o inibidor

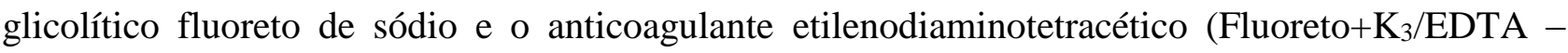
Labor Import $^{\circledR}$ ), centrifugadas a 250 xg por 10 minutos (centrífuga Excelsa Baby II 206-R Fanem $^{\circledR}$ ) e processadas por meio do método bioquímico (Glicose Liquiform - Labtest ${ }^{\circledR}$ ) com leitura fotométrica (analisador bioquímico Bio-2000 - Bio Plus ${ }^{\circledR}$ ) do qual se obteve o valor glicêmico de referência.

Para as mensurações em glicosímetro, utilizaram-se alíquotas das mesmas amostras de sangue central, porém sem anticoagulante, conforme orientações do fabricante. O aparelho utilizado foi o digital portátil On-Call ${ }^{\circledR}$ Plus (Acon Laboratories ${ }^{\circledR}$ ), projetado para pacientes humanos. Esse aparelho é indicado para mensurar sangue capilar e utiliza o sistema amperométrico glicose-oxidase dependente, com necessidade de $1 \mu \mathrm{L}$ de sangue para avaliação, cujo resultado é fornecido em 10 segundos. No sistema de medição amperométrico, a tira-teste reagente inserida no aparelho em contato com a amostra de sangue sofre reação eletroquímica, em que a intensidade da corrente elétrica resultante é proporcional à concentração de glicose na amostra, sendo assim, avaliada pelo medidor que determina o resultado em $\mathrm{mg} / \mathrm{dL}$.

Após colheita venosa central, procedeu-se à realização de punção na face interna do pavilhão auricular do animal para extração de gota de sangue capilar, também posteriormente avaliada no glicosímetro, apresentando o resultado do valor glicêmico capilar. Os dados foram avaliados por meio da análise descritiva dos dados para obtenção das médias e desvios-padrão. Sequencialmente, foram submetidos à Análise de Variância (ANOVA) "one way", e ao pós-teste Holm Sidask's, utilizando-se para isto o programa de análise estatística GraphPad Prism ${ }^{\circledR}$ 6. Em todos os casos, o nível de significância foi de $\mathrm{p} \leq 0,05$. 


\section{Resultados e Discussão}

Para os 20 animais do estudo, a concentração de glicose mensurada pelo glicosímetro nas amostras de sangue capilar variou de 50 a $98 \mathrm{mg} / \mathrm{dL}$. Nas avaliações das amostras de sangue venoso central, também obtidas pelo aparelho portátil, os valores glicêmicos foram de 71 a $125 \mathrm{mg} / \mathrm{dL}$. Pelo método laboratorial de referência, que utilizou sangue venoso central, constatou-se valores de 63 a $109 \mathrm{mg} / \mathrm{dL}$. As médias alcançadas pelos métodos utilizados e seus respectivos desvios-padrão estão representadas na Tabela 1 .

Tabela 1: Médias e desvios padrão dos valores glicêmicos, obtidos através de dois diferentes métodos de mensuração

\begin{tabular}{cccc}
\hline & GSC (mg/dL) & GSVC (mg/dL) & LPVC (mg/dL) \\
\hline Média & 73,1 & 84,1 & 80 \\
Desvio-padrão & $\pm 11,0$ & $\pm 11,7$ & $\pm 10,9$ \\
\hline GSC: Glicosímetro com Sangue Capilar & & \\
GSVC: Glicosímetro com Sangue Venoso Central & & \\
LPVC: Método Laboratorial com Plasma Venoso Central & &
\end{tabular}

$\mathrm{Na}$ análise comparativa houve diferença significativa $(\mathrm{p}<0,05)$ entre os métodos e procedência das amostras. Ou seja, os valores glicêmicos obtidos pelo glicosímetro versus o método laboratorial apresentaram significância estatística, assim como os valores de glicemia obtidos pelo aparelho com as distintas amostras sanguíneas. Além disso, foi observada variação inferior a 15 ou $20 \%$ entre as médias pelos diferentes métodos (Tabela 2), como recomendado por instituições reguladoras de glicosímetros e por entidade de padronização internacional.

Tabela 2: Variação e índice de significância entre os métodos de análise glicêmica

\begin{tabular}{ccc}
\hline & Variação (\%) & p \\
\hline GSC e LPVC & 8,6 & 0,0120 \\
GSVC e LPVC & 5,1 & 0,0405 \\
GSC e GSVC & 13,07 & $<0,0001$ \\
\hline
\end{tabular}

GSC: Glicosímetro com Sangue Capilar

GSVC: Glicosimetro com Sangue Venoso Central

LPVC: Método Laboratorial com Plasma Venoso Central

Amostras de sangue capilar mensuradas por meio do glicosímetro resultaram em média glicêmica inferior à obtida pelo método em laboratório com plasma venoso central. Essa diferença pode ocorrer devido à natureza densa do sangue pelas hemácias, pois a concentração de glicose no sangue total é aproximadamente 10 a $15 \%$ menor do que no plasma, o qual é utilizado para análise bioquímica laboratorial $^{(14)}$. Entretanto, ao se comparar o método glicosímetro utilizando sangue venoso central versus o método em laboratório, em que se utilizou sangue proveniente da mesma amostra, a média glicêmica pelo glicosímetro foi superior, corroborando o estudo de Santos et al. ${ }^{(5)}$, que utilizou sangue de cães. Possivelmente, isso ocorreu porque a amostra avaliada pelo glicosímetro foi imediatamente mensurada, enquanto que o sangue em EDTA e fluoreto foi 
encaminhado ao laboratório para centrifugação e posterior processamento bioquímico. Embora se tenha usado o fluoreto (redutor do metabolismo glicolítico), é possível que alguma porcentagem de glicose tenha sido metabolizada no período até o processamento laboratorial, o que gerou pequena diferença na comparação entre a glicemia venosa central mensurada pelo glicosímetro e a glicemia mensurada em laboratório. Tal afirmação é justificada pelos achados de Ferreira et al. ${ }^{(1)}$, que afirmaram que pode haver decréscimo de 5 a $7 \%$ da glicose já na primeira hora, semelhante à variação observada neste estudo $(5,1 \%)$.

Ao se analisarem os valores obtidos pelo glicosímetro, comparando-se amostras de sangue venoso central e periférico, observou-se que a média glicêmica com sangue capilar foi inferior quando comparada à média com sangue venoso central. Resultados semelhantes em gatos foram observados por Wess e Reusch ${ }^{(7)}$, Thompson et al. ${ }^{(15)}$ e Fracassi et al. ${ }^{(16)}$. A diferença pode ocorrer devido à influência da pressão parcial de oxigênio $\left(\mathrm{pO}_{2}\right)$ nos glicosímetros, que funcionam por sistema amperométrico glicose-oxidase dependente, como explicaram Wess e Reusch ${ }^{(7)}$. Segundo os autores, o sangue capilar possui maior $\mathrm{pO}_{2}$ do que o sangue venoso e o oxigênio compete por elétrons na reação eletroquímica que ocorre no modo de funcionamento de tais aparelhos. Quando há elevada $\mathrm{pO}_{2}$ na amostra, há maior competição do oxigênio durante a reação e menos elétrons são formados ao final, o que reflete na redução da intensidade da corrente elétrica e, consequentemente, redução do resultado glicêmico, já que a intensidade da corrente elétrica é que determina o resultado ${ }^{(13,17)}$. Da mesma forma que a elevada $\mathrm{pO}_{2}$ pode subestimar a glicemia, a reduzida $\mathrm{pO}_{2}$ na amostra pode elevar o resultado ${ }^{(13)}$. Tais afirmações podem explicar os resultados encontrados no presente estudo, em que sangue capilar e venoso central foram mensurados por glicosímetro do tipo amperométrico glicose-oxidase dependente e apresentaram média glicêmica menor e maior, respectivamente. Dessa forma, uma vez que a influência do oxigênio existe, esse tipo de efeito merece atenção e não se recomenda a utilização de tais aparelhos em pacientes críticos ou em oxigenoterapia $^{(13,17,18)}$.

Ademais, existem outros fatores que alteram o valor glicêmico, entre eles o volume globular (VG) do paciente. Animais com baixo VG podem ter a glicemia superestimada pelo aparelho ${ }^{(4,6)}$. Acredita-se que este fato se deva ao aumento da difusão do plasma na área reagente da fita, já que há menor volume de hemácias ocupando esta área, e o plasma possui concentração de glicose 10 a $15 \%$ maior do que o sangue total ${ }^{(4,14)}$. O volume da amostra é outro fator pertinente, pois quando quantidade insuficiente de sangue é absorvida pela tira-teste do aparelho, a depender do modelo, este pode não notificar o erro em seu monitor e fornecer resultado inferior para a glicemia ${ }^{(16)}$. O jejum é outro fator importante a ser considerado, pois, como observado em estudos com humanos, no período pós-prandial o valor glicêmico no sangue capilar pode estar até $7 \%$ maior do que o observado no sangue venoso, devido à proximidade dos capilares com as artérias, as quais possuem maiores concentrações do carboidrato ${ }^{(13,19)}$. No entanto, como este estudo foi desenvolvido com animais em jejum, não foram observados níveis superiores da glicemia no sangue capilar em relação ao venoso central.

As divergências entre os resultados alcançados pelos diferentes métodos são aceitáveis, visto que estão dentro dos limites estabelecidos por associações reguladoras de glicosímetros, como a $A D A$ e a $F D A$, que preconizam variação de até $15 \%$ e $20 \%$, respectivamente, na concentração glicêmica para valores entre 30 e $400 \mathrm{mg} / \mathrm{dL}^{(14)}$; e do que é recomendado pela $I S O$, que padronizou variação de até $20 \%$ entre valores glicêmicos observados por diferentes métodos de mensuração em relação aos obtidos pelo método laboratorial ${ }^{(10,11)}$. 
Existem muitos debates científicos acerca da acurácia dos mensuradores portáteis de glicose $^{(6,12,15,16)}$. Estudos recentes avaliaram uma série de aparelhos que apresentaram resultados próximos aos obtidos pelo método laboratorial, porém, alguns modelos demonstram ser mais precisos do que outros $^{(1)}$. Assume-se que não seja necessário debater qual glicosímetro seja o mais acurado e preciso caso atenda às necessidades clínicas para o controle da glicemia, com variação percentual dos valores encontrados em relação ao laboratório abaixo da recomendada por associações reguladoras dos aparelhos e de padronização internacional. Adicionalmente, como observado neste estudo, o glicosímetro amperométrico apresentou valores dentro do intervalo de variação estabelecido pelos órgãos reguladores, inclusive com variação próxima a 5\% na comparação entre a glicemia venosa central mensurada pelo aparelho e a técnica laboratorial.

A mensuração da glicemia capilar pelo glicosímetro foi realizada em amostra de sangue puncionado na face interna da orelha dos felinos, procedimento que se revelou de fácil realização. O uso desta região para punção minimiza o desconforto do paciente e preserva a integridade de veias periféricas ${ }^{(15)}$. Assim como observado neste estudo, esta técnica tem sido utilizada com sucesso na espécie felina e pode ser satisfatoriamente realizada em domicílio ${ }^{(3)}$. No entanto, Casella et al. ${ }^{(1)}$ observaram que uma fase de treinamento do proprietário é necessária para a adequada realização do exame. Ao longo do tempo, os animais se tornam cada vez mais tolerantes às punções nesta região da orelha e o êxito pode ser obtido após certo período de adaptação ${ }^{(20)}$. Além disso, apesar de simples, observou-se que a punção na face interna da orelha dos animais pode ser auxiliada por feixe de luz posicionado atrás da região e também por fricção local para aumento da irrigação sanguínea, permitindo melhor visualização dos capilares.

O uso de sangue capilar ou a partir de vasos da periferia torna-se inviável quando os pacientes encontram-se em estado de choque, desidratação profunda ou reduzida vascularização periférica ${ }^{(6)}$, o que torna o procedimento desvantajoso em relação ao uso de sangue venoso proveniente da jugular, até que o volume da circulação periférica seja restabelecido. Além disso, o uso da via capilar em pacientes hemodinamicamente instáveis pode levar a resultados falsos da glicemia e refletir em inadequado protocolo terapêutico ${ }^{(21)}$. Desse modo, é importante se atentar para a via de coleta ideal para mensuração glicêmica em pacientes críticos.

Já para os pacientes internados e com acesso venoso, especialmente com cateteres centrais, pode-se sugerir a utilização de amostragem coletada a partir desses dispositivos e mensuração em glicosímetro portátil, uma vez que a diferença foi de apenas $5,1 \%$ em comparação aos valores obtidos pelo laboratório. Dessa forma, obtém-se resultados mais rápidos e os custos do procedimento são minimizados. Tal conduta torna-se mais relevante considerando-se que animais internados são periodicamente monitorados para outros parâmetros, permitindo que, da mesma amostra sanguínea coletada para outros exames, seja utilizada pequena alíquota para obtenção da glicemia pelo glicosímetro portátil com resultado imediato.

\section{Conclusões}

Os resultados evidenciaram que o glicosímetro utilizado no estudo pode ser indicado no monitoramento eficaz da glicemia em felinos como alternativa ao método laboratorial, usando-se tanto amostras de sangue venoso central como de sangue capilar. 


\section{Agradecimentos}

Aos profissionais e estagiários do Hospital Veterinário de Pequenos Animais e do Laboratório de Patologia Clínica Veterinária da Universidade de Brasília.

\section{Comissão de ética}

A pesquisa obteve aprovação pela Comissão de Ética no Uso de Animais (CEUA) do Instituto de Ciências Biológicas da Universidade de Brasília, protocolado com o número 42871/2012.

\section{Referências}

1. Casella M, Wess G, Reusch CE. Measurement of capillary blood glucose concentrations by pet owners: a new tool in the management of diabetes mellitus. Journal of the American Animal Hospital Association. 2002;38(3):239-245. Disponível em: http://www.ncbi.nlm.nih.gov/pubmed/12022410

2. Casella M, Wess G, Hässig M, Reusch CE. Home monitoring of blood glucose concentration by owners of diabetic dogs. Journal of Small Animal Practice. 2003;44(7):298-305. Disponível em: http://onlinelibrary.wiley.com/doi/10.1111/j.1748-5827.2003.tb00158.x/abstract

3. Reusch CE, Kley S, Casella M. Home monitoring of the diabetic cat. Journal of Feline Medicine and Surgery. 2006;8(2):119-127. Disponível em: http://www.sciencedirect.com/science/article/pii/S1098612X05001518

4. Stein ES, Greco DS. Portable blood glucose meters as a means of monitoring blood glucose concentrations in dogs and cats with diabetes mellitus. Clinical Techniques in Small Animal Practice. 2002;17(2):70-72. Disponível em: http://lib.gen.in/f4b98c6891c6cf9f159acb0eba0d3674/stein2002.pdf

5. Santos TF, Rubio MS, Neves ETV, Silva JES, Guerra CRSB, Coelho WMD, et al. Avaliação comparativa entre o glicosímetro portátil e o método laboratorial enzimático colorimétrico na dosagem glicêmica em cães. Ciências Agrárias e da Saúde. 2008;8:7-11. Disponível em: http://www.fea.br/Arquivos/Revista\%20Cientifica/Volume\%208\%202008.pdf

6. Wess G, Reusch C. Evaluation of five portable blood glucose meters for use in dogs. Journal of the American Veterinary Medical Association. 2000a;216(2):203-209. Disponível em: http://avmajournals.avma.org/doi/abs/10.2460/javma.2000.216.203

7. Wess G, Reusch C. Capillary blood sampling from the ears of dogs and cats and use of portable meters to measure glucose concentration. Journal of Small Animal Practice. 2000b;41(2):60-66. Disponível em: http://onlinelibrary.wiley.com/doi/10.1111/j.1748-5827.2000.tb03164.x/abstract

8. Aleixo GAS, Coelho MCOC, Guimarães ALN, Andrade MB, Silva JAA. Avaliação comparativa entre o glicosímetro portátil e o método laboratorial enzimático-colorimétrico segundo Trinder na dosagem glicêmica em cães. Revista Portuguesa de Ciências Veterinárias. 2007a;102(563-564):351-354. Disponível em: http://www.fmv.utl.pt/spcv/PDF/pdf12_2007/351-354.pdf

9. Aleixo GAS, Coelho MCOC, Tenório APM, Silva JAA, Guimarães ALN, Andrade MB, et al. Mensuração da glicemia em cães mediante a utilização do glicosímetro portátil: comparação entre amostras de sangue capilar e venoso. Medicina Veterinária. 2007b;1(1):9-13. Disponível em: 


\section{http://snida.agricultura.gov.br:81/binagri/bases/agb/Agb_Docs_Fonte/BR0704837.pdf}

10. Aleixo GS, Coelho MCOC, Tenório APM, Guimarães ALN, Andrade MB, Cavalcanti HB. Uso do glicosímetro portátil para determinar a concentração de glicose no sangue de cães. Ciência Animal Brasileira. 2010;11(3):537-545. Disponível http://h200137217135.ufg.br/index.php/vet/article/view/3700/7919

11. Ferreira PA, Mueller EM, Fischer EC, Tillmann MT, Peres W, Nobre MO. Glicemia do sangue capilar e venoso de cães saudáveis: mensuração por método eletroquímico versus enzimático laboratorial. Semina: Ciências Agrárias. 2013;34(3):1287-1292. Disponível em: http://www.uel.br/revistas/uel/index.php/semagrarias/article/view/12824/pdf

12. Hönes J, Müller P, Surridge N. The technology behind glucose meters: test strips. Diabetes Technology \& Therapeutics. 2008;10(1):10-26. Disponível em: http://online.liebertpub.com/doi/abs/10.1089/dia.2008.0005

13. Ginsberg BH. Factors affecting blood glucose monitoring: sources of errors in measurement. Journal of Diabetes Science and Technology. 2009;3(4):903-913. Disponível em: http://www.glucorx.co.uk/wpcontent/uploads/2012/02/Factors-effecting-Blood-Glucose-Monitoring-2.pdf

14. Briggs AL, Cornell S. Self-monitoring blood glucose (SMBG): now and the future. Journal of Pharmacy Practice. 2004;17(1):29-38. Disponível em: http://jpp.sagepub.com/content/17/1/29.short

15. Thompson MD, Taylor SM, Adams VJ, Waldner CL, Feldman, EC. Comparison of glucose concentrations in blood samples obtained with a marginal ear vein nick technique versus from a peripheral vein in healthy cats and cats with diabetes mellitus. Journal of the American Veterinary Medical Association. 2002;331(3):389-392. Disponível em: http://europepmc.org/abstract/MED/12164536

16. Fracassi F, Hadar, GS, Pietra M, Famigli, BP. Assessment of two portable blood glucose meters for use in cats and dogs. Journal of Veterinary Clinical Sciences. 2009;2(4):108-121.

17. Tang Z, Louie RF, Lee JH, Lee DM, Miller EE, Kost GJ. Oxygen effects on glucose meter measurements with glucose dehydrogenase- and oxidase-based test strips for point-of-care testing. Critical Care Medicine. 2001;29(5):1062-1070. Disponível em: http://cat.inist.fr/?aModele=afficheN\&cpsidt=989148

18. Freckmann G, Schmid C, Baumstark A, Pleus S, Link M, Haug C. Partial pressure of oxygen in capillary blood samples from the fingertrip. Journal of Diabetes Science and Technology. 2013;6(7):1648-1649. Disponível em: http://jdst.org/November2013/PDF/VOL-7-6-LTE3-FRECKMANN.pdf

19. Yang C, Chang C, Lin J. A comparison between venous and finger-prick blood sampling on values of blood glucose. International Proceedings of Chemical, Biological and Environmental Engineering. 2012;39:206-210. Disponível em: http://www.ipcbee.com/vol39/040-ICNFS2012-N3003.pdf

20. Van de Maele I, Rogier N, Daminet S. Retrospective study of owners' perception on home monitoring of blood glucose in diabetic dogs and cats. The Canadian Veterinary Journal. 2005;46(8):718-723. Disponível

em:

https://biblio.ugent.be/input/download?func=downloadFile\&recordOId=378413\&fileOId=789904

21. Argollo APB; Faustino TN; Faustino TN; Pedreira LC. Valores glicêmicos oferecidos pelo glicosímetro portátil, utilizando sangue de diferentes vias de coleta: estudo de validade. Revista Brasileira de Terapia Intensiva. 2010;4(22):351-357. Disponível em: http://www.scielo.br/pdf/rbti/v22n4/07.pdf 